\title{
Self-standing Nanoparticle Membranes and Capsules
}

\author{
Henry Chan and Petr Král* \\ Department of Chemistry, University of Illinois at Chicago, Chicago, IL 60607, USA
}

(Dated: May 19, 2011)

\begin{abstract}
We perform coarse-grained molecular dynamics simulations of self-standing nanoparticle membranes observed in recent experiments (Nat. Mater. 6, 656 (2007)). In order to make our simulations feasible, we model $2-3$ times smaller gold nanoparticles (core radius of $r_{\text {core }} \approx 0.8 \mathrm{~nm}$ ) covered with alkanethiol ligands (length of $l_{\text {ligand }} \approx 0.5-2.6 \mathrm{~nm}$ ). We study the structure, stability, and mechanical properties of these membranes and show that these characteristics are controlled by the ratio of $R_{L C}=l_{\text {ligand }} / r_{\text {core }}$. For $R_{L C} \approx 0.6$, the ligated nanoparticles form well ordered monolayers with hexagonal packing, in agreement with the experiments $\left(R_{L C} \approx 0.44\right)$. For $R_{L C} \approx 1.6$, the nanoparticles form less organized multilayers, which are more stable and flexible. We show that these membranes could potentially form stable capsules for molecular storage and delivery.
\end{abstract}


In recent years, colloidal nanoparticles (NP) were self-assembled into many types of superlattices $[1,2]$, with packing controlled by forces originating from the NP bulk, ligands, and solvent. It turns out that ligands can play an important role in controlling the NP packing. Even simple monodisperse gold NPs covered by alkylthiols, can form bulk fcc and bcc superlattices for $R_{L C}<0.8$ and $R_{L C}>0.8$, respectively, where $R_{L C}=l_{\text {ligand }} / r_{\text {core }}$ is the ratio of the NP core radius, $r_{\text {core }}$, and the ligand length, $l_{\text {ligand }}[3]$. The anisotropic forces responsible for this fcc-bcc transition observed at larger $R_{L C}$ originate in the self-organization of ligands, which form bundles, and tend to arrange NPs in linear chains $[4,5]$. Monolayers of colloidal NPs have also been formed on solid substrates $[6,7]$ and solvent interfaces [8, 9]. Their electrical [10-12] and optical properties [13, 14] of depend on the NP type, size, ligands [15], and substrate used $[16,17]$.

Recently, highly ordered self-standing monolayers with hexagonal packing have been assembled from gold NPs with the core radius of $r_{\text {core }} \approx 3 \mathrm{~nm}$ and coated with dodecanethiol ligands of the length of $l_{\text {ligand }} \approx 1.3 \mathrm{~nm}\left(R_{L C} \approx 0.44\right)$ [9]. The monolayers were formed by the drop-drying method on the surface of a toluene droplet, and removed by evaporating the solvent. AFM indentation experiments have shown that these membranes remain elastic up to $T=370 \mathrm{~K}$ [18], and that their mechanical strength depends on the types of NPs used [19]. The NP-membranes have potential applications in electronics [20-22], molecular filtering, sensing [23], and delivery.

In this work, we perform the first microscopic modeling of the self-standing membranes and capsules formed by self-assembled gold NPs covered with alkanethiol ligands. We study the structure, the stability, and the mechanical properties of these large systems by coarsegrained molecular dynamic simulations (CGMD) with the Martini 2.0 force field [24], implemented in the NAMD package [25, 26].

In Fig. 1 (a-d), we show the CG models of our small ligated gold NPs, with the core radius of $r_{\text {core }} \approx 0.8 \mathrm{~nm}$, which is $\approx 3-4$ times smaller than NP-cores in the experimentally studied NP-membranes $[9,27]$. Smaller NPs allow us to simulate the membrane dynamics with current computational resources. From the point of mechanical properties, membranes with larger NPs, used in experiments, could be roughly mapped on our model systems, by keeping similar $R_{L C}$ and scaling the ligand-ligand coupling strengths.

We represent gold atoms in the NPs by $\mathrm{SC}_{4}$-type beads [24], where each bead approximately 
corresponds to four gold atoms. We assign new parameters to these $\mathrm{SC}_{4}$-type beads, because gold atoms are not included in the Martini force field. The $\mathrm{SC}_{4}-\mathrm{SC}_{4}$ bond distance matches the lattice constant of the gold atoms $(4.08 \AA)$, while the other parameters, such as the bond strength $(15.0 \mathrm{kcal} / \mathrm{mol})$ and the $\mathrm{SC}_{4}-\mathrm{SC}_{4}-\mathrm{SC}_{4}$ angle $\left(60^{\circ}, 2.988 \mathrm{kcal} / \mathrm{mol}\right)$, are chosen to give a rigid core. The $\mathrm{SC}_{4}$ beads are arranged into $f c c$ close packing, with bonding distances of $\approx 0.4 \mathrm{~nm}$ forming the cuboctahedral NP-core. Very small gold clusters are preferentially formed with five-fold (icosahedron) symmetries [28, 29], but gold NPs with different core geometries have similar properties [30]. The core structure is less relevant in our studies, especially for longer ligands. For short ligands, the shape and facets of the gold cores could play a role in a local organization of the NP-superlattices, particularly at low temperatures. The NP-cores are capped with alkanethiol ligands with the density of 1 ligand per each surface gold bead $\left(\approx 15.4 \AA^{2}\right.$ per ligand). The ligands are represented by nonpolar $\mathrm{C}_{1}$-type beads, giving 3 beads for dodecanethiol, as shown in Fig. 1 (e). The ligands used in the simulations have 1 to $6 \mathrm{C}_{1}$-type beads, as shown in Fig. 1 (a-d). We label the NP covered with $n$-beads ligands as the $n$-bead NP. Ligands of different coupling strengths are created by replacing some of the $\mathrm{C}_{1}$-type beads by $\mathrm{C}_{2}$-type beads [24], and defining the $\mathrm{C}_{1}-\mathrm{C}_{2}$ and $\mathrm{C}_{2}-\mathrm{C}_{2}$ van der Waals (vdW) coupling strengths.

Bonded interactions between the beads are defined in the Martini force field [24], where the non-bonded vdW interactions are described by the Lennard-Jones potential,

$$
V_{L J}(i, j)=\epsilon_{i j}\left\{\left(\frac{\sigma_{i j}}{r_{i j}}\right)^{12}-\left(\frac{\sigma_{i j}}{r_{i j}}\right)^{6}\right\} .
$$

Here, $i, j$ are non-covalently bonded beads, $\epsilon_{i j}$ is the depth of the potential well (strength of interaction), $\mathrm{r}_{i j}$ is the distance between bead $i$ and $j$, and $\sigma_{i j}$ is the closest distance of approach between bead $i$ and $j$. For free-standing membranes in vacuum, particle core-core vdW interactions are typically small, in comparison to ligand-ligand vdW interactions.

Nanoparticle membranes. When a certain number of NPs are put in contact with each other, they spontaneously form a cluster of more or less spherical shape. To prepare membrane monolayers, we arrange 91 NPs, with the chosen length of ligands, close to each other (their ligands overlap) into a hexagonal lattice confined in a plane. This planar cluster has the shortest boundary and its edge energy (absence of bonding) is minimized. If the NPs are constrained in a plane and placed close to each other, they can also spontaneously form 
this hexagonal cluster at $t>100$ ns.

We confine the NPs in the $z=0$ plane by applying to the central bead in each of them the force of

$$
\vec{F}_{\text {plane }}=-k_{1} \vec{z} /|\vec{z}|
$$

where we choose a large $k_{1} \approx 200 \mathrm{pN}$. We simulate the NPs for $30 \mathrm{~ns}$ and in the end do not observe any waving of the NPs out of the $z=0$ plane at this $k_{1}$. The NP-NP distances are spontaneously optimized by the vdW coupling of their ligands (enthalpic and entropic contributions). Then, the central beads of NPs on the edge of the membrane are fixed, $\vec{F}_{\text {plane }}$ is removed, and the membranes are relaxed for another 30 ns. If this stabilization is done at small $k_{1}$ parameters, the NPs would not allign in the plane enough and the next step (stretching) would start from a multilayer system. All the simulations are performed in the NVT ensemble with periodic boundary conditions in vacuum. The temperature of $T=310$ $\mathrm{K}$ is maintained by Langevin damping, with the damping coefficient of $\gamma_{\text {Lang }}=1 \mathrm{ps}^{-1}$, and the simulation timestep is $20 \mathrm{fs} / \mathrm{step}$.

As shown in Fig. 2 (a-c), obtained for the 1 -3-bead NPs, respectively, the relaxed membranes with fixed border NPs reorganize into more or less perturbed planar structures. The 1-bead NPs $\left(R_{L C}=0.62\right)$ form wavy monolayers, the 2-bead NPs $\left(R_{L C}=1.12\right)$ sometimes form partial double-layers (2 figures shown), while 3-bead NPs $\left(R_{L C}=1.62\right.$ ) always form partial double-layers. The morphology of the membranes is determined by the ligand type and the initial stretching. In short-haired NPs, the ligands just provide coupling of the cluster cores that dominate the whole structure. The 1-bead NPs tend to rotate more, which can average out any potential effects due to the core shape. These membranes are highly organized and form monolayers, in analogy to the experimental systems $\left(R_{L C} \approx 0.44\right)[9]$. On the other hand, the morphology of membranes with long-haired NPs is mostly controlled by the ligands (negligible core size). These membranes are less organized, form multilayers, and behave like short (cross-linked) polymers. The stability and strength of the three types of membranes is also different, and given by the ligand interdigitation (vdW coupling).

The NPs in the membranes have fast rotational motion, but very slow translational lateral diffusion, practically absent in the stretched membranes. In Fig. 3, we show random angular rotation in the azimuthal angular direction of the NP located in the center of the relaxed (non-stretched) membranes. It turns out that the NP rotation rate dramatically decreases 
with $R_{L C}$. The 1 -bead NP rotates by $180^{\circ}$ in 30 ns, the 2 -bead NP turns from time to time by $90^{\circ}$, and the 3 -bead NP is almost static. The 1 -bead NP has almost $180^{\circ}$ inversion in $27 \leq t \leq 36 \mathrm{~ns}$, which results in a sharper transition and indicates that reorientation takes place sooner than NP with longer ligands.

We stretch the NP-membranes by a "virtual probe" that locally pushes on the membranes, in analogy to an AFM. The probe is defined by the stretching force, applied to the central bead of every NP, oriented in the $z$-direction, and having the $(x, y)$-profile

$$
\vec{F}_{\text {stretch }}=-k_{2} \exp \left(-\left(x^{2}+y^{2}\right) / 2 c^{2}\right) \vec{z} /|\vec{z}|
$$

Here, $c=\sqrt{21}=4.58 \mathrm{~nm}$ is the effective half-width of the probe, and $k_{2}$ is the force magnitude. $c$ is chosen to produce a "virtual tip" that is not too sharp to easily penetrate the membrane, which happens at $c \geq \sqrt{5} \mathrm{~nm}$. It should not be too flat for the used membrane, in order to avoid a significant "collision" (force applied to it) with the fixed border of the membrane, which happens at $c \geq \sqrt{50}$. The probe is positioned in the center of the membrane.

We study how the NP-membranes behave in stretch-release cycles going in upward and downward directions. The stress is measured by the average force, $\vec{F}_{\text {stretch }}$, per unit area of the membrane, and the strain is measured by the average $z$-displacement relative to the $z=0$ plane.

$$
D_{z}=\frac{1}{N R_{m}} \sum_{i=1}^{N} d_{z i}
$$

Here, $d_{z i}$ is the average displacement of the $i$ th NP $(N=91)$, and $R_{m} \approx 11 \mathrm{~nm}$ is the membrane radius.

In Fig. 4, we show the stress-strain curves for the membranes in Fig. 2 (a-c), with $R_{L C}=$ $0.62,1.12$ and 1.62. The force constant $k_{2}$ in Eq. 3 is increased or decreased in steps of $69.5 \mathrm{pN}$, and after each step the system is equilibrated for $\approx 20 \mathrm{~ns}$. Then, we calculate the membrane stretching by averaging it over a trajectory of the length of $\approx 20 \mathrm{~ns}$. At low stresses, the strain of the NP-membranes increases linearly with the stress, according to the Hooke's law, with the slope of 38.1, 54.3, 120.6, and $98.0 \mathrm{pN} / \mathrm{nm}^{2}$ (relative displacement) for the 1-bead, 2-bead (monolayer), 2-bead (multilayer), and 3-bead, respectively. At larger stresses, $\sigma>25 \mathrm{pN} / \mathrm{nm}^{2}$, the strain deviates from the linear dependence, and the membranes eventually rupture without recovery. In general, membranes with larger $R_{L C}$ can be stretched 
more. Figure 2 (d-f) shows the 1-3-bead membrane in their half-stretched and fully stretched states.

The stretched 1-bead membrane is always in the form of a monolayer. The 2-bead membrane switches between a monolayer and partial double-layer, giving two different stressstrain dependencies. The formation of double-layers in the 2-3-bead membranes causes the appearance of hysteresis loops in their stress-strain curves, reflecting memory effects.

Nanoparticle capsules. Next, we investigate the possibility of preparing stable capsules from the ligated NPs. In the recent past, novel routines have been designed to prepare microsized and nanosized NP-capsules, suitable for molecular storage and delivery [31]. For example, microsized spherical cavities has been assembled using CdSe nanoparticles at polymer solution-water interfaces [32], and well-defined nanocapsules have been prepared in emulsions [33].

We prepare highly elastic and potentially self-healing capsules from the NPs with 3-bead and 6-bead ligands. The NP-capsules are formed by assembling 96 NPs on a spherical (hard-core) potential surface of a variable radius. The potential is defined by the force

$$
\vec{F}_{H S}=\theta\left(R_{s}-R\right) k_{3} \vec{R}_{0}
$$

acting on the central bead in each NP, which mimics the presence of molecules stored in the capsules. Here, $\theta$ is the Heaviside step function $\left(\theta=1\right.$ when $R<R_{s}, \theta=0$ elsewhere), $R_{s}$ is the variable radius of the potential surface, $k_{3} \approx 700 \mathrm{pN}$ is the magnitude of the applied force, and $\overrightarrow{R_{0}}$ is a unit vector oriented along the direction of the position vector $\vec{R}=(x, y, z) . k_{3}$ is chosen to be sufficiently large to make the sphere rigid. For smaller $k_{3}$, the NPs submerge into it, depending on their stretching $\left(R_{s}\right)$.

In Fig. 5, we show capsules formed by NPs with 3-bead (a-b) and 6-bead (c-d) ligands. Each NP-capsule is prepared by inserting a potential surface of a small $R_{s}$ inside a NP-cluster and by slowly increasing its radius in steps of $0.1 \mathrm{~nm}$, followed by equilibration for $22 \mathrm{~ns}$. After each equilibration, we average the number of NP-neighbors and the NP-distances over all NPs in additional trajectories of the length of $20 \mathrm{~ns}$ to obtain the distributions shown in Fig. 6. The capsule radius is changed in the interval of $R_{s}=5-10 \mathrm{~nm}$, with the step size of $0.1 \mathrm{~nm}$, and the obtained plots are combined to form 2D maps.

The 3-bead capsule has partially a double-layer for $R_{s}=5 \mathrm{~nm}$, as seen in Fig. 5 (a), in 
analogy to the NP-membrane in Fig. 2 (c). With only 96 NPs, we do not observe a complete double-layer capsule even at $R_{s}<5 \mathrm{~nm}$. If we increase the capsule size to $R_{s}=7 \mathrm{~nm}$, it forms a monolayer, as seen Fig. 5 (b). When we further increase $R_{s}$, the coupling strength of the ligands is eventually exceeded, and the capsule ruptures. The capsule with 6-bead ligands is highly stretchable. As we stretch it, the ligands become highly elongated and the NPs more separated, thus covering only a small part of the capsule surface, as shown in Fig. $5(\mathrm{c}-\mathrm{d})$.

We characterize the structure of NP-capsules by investigating the spatial correlations between NPs with different ligands and under different stretching. In Fig. 6 (top) and (bottom), we show for the capsule with the 3-bead ligands the number of first neighbors of a given NP and the distances to all the other NPs.

In planar membranes, NPs with small $R_{L C}$ arrange in a close-packed hexagonal pattern, where each NP has 6 first neighbors. On curved surfaces, the number of first neighbors varies. In the NP-capsules, we define first neighbors as NPs that are within the chosen cutoff distance of $3.7 \mathrm{~nm}$, based on the NP-correlations in Fig. 6 (bottom). As shown in Fig. 6 (top), when the capsule is a monolayer $\left(R_{s}>6.5 \mathrm{~nm}\right), 5$ neighbors $\left(R_{s}>7.3 \mathrm{~nm}\right)$ and 6 neighbors $\left(R_{s}<7.2 \mathrm{~nm}\right)$ predominate. This situation is analogous to $\mathrm{C}_{60}$ fullerene formed by pentagons and hexagons. We can obtain the approximate structure of such capsules if we replace every atom in $\mathrm{C}_{60}$ by a NP and add one more NP in the center of every pentagons and hexagons. When the capsule is formed by a partial double-layer $\left(R_{s}<6.4 \mathrm{~nm}\right)$, the number of neighbors becomes less defined, with more neighbors present due to the second layer.

In Fig. 6 (bottom), we show a 2D map of the average NP distances for different capsule sizes. When the capsule is a monolayer $\left(R_{s}>6.5 \mathrm{~nm}\right)$, we can see clear trend lines for the first 8 neighbors. All the NP separations linearly increase with $R_{s}$. Once a partial double-layer is formed on the NP-capsule $\left(R_{s}<6.5 \mathrm{~nm}\right)$, the trend lines become less defined, occasionally breaking into several lines, and the NP separation almost does not depend on $R_{s}$.

In Fig. 7 (top), we show the same for the NP-capsule with 6-bead ligands. Here, we can see well the trend lines for the first few NP neighbors and only for $R_{s} \approx 9-10 \mathrm{~nm}$, where the membrane is highly stretched. In this state, the ligands are fully extended and the cores are no longer able to move around. For smaller $R_{s}<9$, the 2D map has irregular splittings and 
the NPs do not have well defined separation distances. In these capsules, the long ligands can wrap around any NP and form bundles. To enhance coupling of ligands from different NPs, we modify the 6-bead ligands by increasing their tip-to-tip coupling. We change the last bead in each ligand from the $\mathrm{C}_{1}$-type to the $\mathrm{C}_{2}$-type and define the vdW coupling strength of the $\mathrm{C}_{1}-\mathrm{C}_{2}$ and $\mathrm{C}_{2}-\mathrm{C}_{2}$ beads to be 1.5 and 2 times that of the $\mathrm{C}_{1}-\mathrm{C}_{1}$ beads, respectively. This results in more extended NP ligand shells, which leads to the reappearance of the trend lines, as shown in Fig. 7 (bottom).

In order to make our simulations more realistic, we also introduce defects in the NPs. First, we randomly remove a fixed percentile of ligands from the whole capsule with the 3-bead ligands, thus creating a variety of defective NPs with different ligand densities. In Fig. 8 (left), we show the distribution of NP-distances after removal of $10 \%$ of ligands. At this ligand density, the overall structure of the capsule is similar to the defect-free capsule, since the NPs rotate to compensate for their ligand loss in certain regions. However, if we compare the distribution in Fig. 8 (left) with the results obtained in the non-defective capsule, we do not see a well defined transition between the monolayer and double-layer capsules, observed at $R_{s} \approx 6.5 \mathrm{~nm}$ in Fig. 6 (bottom). When $10 \%$ and $20-25 \%$ of ligands are removed, the capsule is stable for $R_{s}<7.8 \mathrm{~nm}$ and $6.2 \mathrm{~nm}$, respectively. When $>33 \%$ of ligands are removed, the capsule is unstable. Therefore, as the number of defects grow the capsules become less organized and less stable.

In Fig. 8 (right), we also study the effect of adding hydrophobic dodecane molecules to the non-defective capsule with 3-bead ligands. Initially the molecules are randomly added to the outer surface of the capsule. The molecules are quickly absorbed between the NPs, since they are similar in nature to the alkanethiol ligands. They act as bridges/linkers that give more freedom to the NPs to adjust their positions. After equilibration, the capsule retains its structural regularity, as reflected by the sharp trend lines. The double-layer region, originally observed at $R_{s}<6.5 \mathrm{~nm}$, is slightly shifted to the left. This shows that the NPcoupling is more relaxed in the presence of dodecane molecules. Moreover, the maximum $R_{s}$ is extended by $\approx 0.5 \mathrm{~nm}$.

The large elasticity and potential self-healing ability of these NP-capsules suggest that they could be applied in molecular storage, transportation, and delivery. The capsules can only store molecules that do not break or solvate the ligated NPs. The nature of alkanethiol gold 
NPs limit the kind of substances that can be put into the capsule. Hydrophobic molecules dissolve the NPs and destroy the capsule. Similarly, alcohol can break the Au-S bond and detach ligands from the NP cores.

We prepare two capsules, consisting of 96 NPs and 384 NPs, filled with coarse-grained water (one bead) [24]. After equilibrating these water-filled capsules, we merge them, as shown in

Fig. 9 (left). The joined structure acquires the shape of matryoska (right). The sectional view shows that a NP-monolayer separates waters in the two compartments. When we raise the temperature from $T=310 \mathrm{~K}$ to $T=370 \mathrm{~K}$, the NP-monolayer breaks down and the two water compartments join each other. This might allow to realize a controlled chemical reaction inside NP-capsules.

We performed the first CGMD simulations of NP-membranes and capsules to investigate their structure, stability, and mechanical properties for NPs with ligands of different lengths. We have shown that material properties of these nanosystems are largely controlled by the $R_{L C}$ ratio. For smaller $R_{L C}<1$, the systems form well organized monolayers that are

relatively fragile and less stretchable. For larger $R_{L C}>1$, the structures are less organized, more stable, and highly elastic. The systems with large $R_{L C}$ might potentially be used to form self-healing membranes and capsules.

\section{Acknowledgment}

We acknowledge Heinrich Jaeger for many fruitful discussions. The calculations were performed at the NERSC, NCSA, and CNM-ANL computer networks.

* The address for correspondence: pkral@uic.edu

[1] S. Coe, W.-K. Woo, M. Bawendi, V. Bulovic, Nature 2002, 420, 800.

[2] E. V. Shevchenko, D. V. Talapin, N. A. Kotov, S. O’Brien, C. B. Murray, Nature 2006, 439, 55.

[3] U. Landman, W. D. Luedtke, Faraday Discuss. 2004, 125, 1.

[4] P. Schapotschnikow, R. Pool, T. J. H. Vlugt, Mol. Phys. 2007, 105, 3177.

[5] P. Schapotschnikow, T. J. H. Vlugt, J. Chem. Phys. 2009, 131, 124705.

[6] H. X. He, H. Zhang, Q. G. Li, T. Zhu, S. F. Y. Li, Z. F. Liu, Langmuir 2000, 16, 3846.

[7] C. Jiang, V. V. Tsukruk, Soft Matter 2005, 1, 334. 
[8] Y. Lin, H. Skaff, A. Böker, A. D. Dinsmore, T. Emrick, T. P. Russell, J. Am. Chem. Soc. 2003, 125, 12690 .

[9] T. P. Bigioni, X.-M. Lin, T. T. Nguyen, E. I. Corwin, T. A. Witten, H. M. Jaeger, Nat. Mater. 2006, 5, 265.

[10] D. Bethell, M. Brust, D. Schiffrin, C. Kiely, J. Electroanal. Chem. 1996, 409, 137.

[11] M. D. Musick, C. D. Keating, M. H. Keefe, M. J. Natan, Chem. Mater. 1997, 9, 1499.

[12] S. Pradhan, J. Sun, F. Deng, S. Chen, Adv. Mater. 2006, 18, 3279.

[13] A. Taleb, C. Petit, M. P. Pileni, J. Phys. Chem. B 1998, 102, 2214.

[14] J. M. Weissman, H. B. Sunkara, A. S. Tse, S. A. Asher, Science 1996, 274, 959.

[15] J. E. Martin, J. P. Wilcoxon, J. Odinek, P. Provencio, J. Phys. Chem. B 2000, 104, 9475.

[16] D. V. Talapin, E. V. Shevchenko, C. B. Murray, A. V. Titov, P. Král, Nano Lett. 2007, 7, 1213.

[17] A. V. Titov, P. Král, Nano Lett. 2008, 8, 3605.

[18] K. E. Mueggenburg, X.-M. Lin, R. H. Goldsmith, H. M. Jaeger, Nat. Mater. 2007, 6, 656.

[19] J. He, P. Kanjanaboos, N. L. Frazer, A. Weis, X.-M. Lin, H. M. Jaeger, Small 2010, 6, 1449.

[20] J. A. Rogers, Y. Huang, Proc. Natl. Acad. Sci. U.S.A. 2009, 106, 10875.

[21] V. L. Pushparaj, M. M. Shaijumon, A. Kumar, S. Murugesan, L. Ci, R. Vajtai, R. J. Linhardt, O. Nalamasu, P. M. Ajayan, Proc. Natl. Acad. Sci. U.S.A. 2007, 104, 13574.

[22] Y. Ding, P. Zhang, Z. Long, Y. Jiang, F. Xu, W. Di, Sci. Technol. Adv. Mat. 2008, 9, 015005.

[23] C. Jiang, S. Markutsya, Y. Pikus, V. V. Tsukruk, Nat. Mater. 2004, 3, 721.

[24] S. J. Marrink, H. J. Risselada, S. Yefimov, D. P. Tieleman, A. H. de Vries, J. Phys. Chem. B 2007, 111, 7812 .

[25] J. C. Phillips, R. Braun, W. Wang, J. Gumbart, E. Tajkhorshid, E. Villa, C. Chipot, R. D. Skeel, L. Kalé, K. Schulten, J. Comput. Chem. 2005, 26, 1781.

[26] W. Humphrey, A. Dalke, K. Schulten, J. Mol. Graphics 1996, 14, 33.

[27] V. Santhanam, J. Liu, R. Agarwal, R. P. Andres, Langmuir 2003, 19, 7881.

[28] K. Bao, S. Goedecker, K. Koga, F. Lanon, A. Neelov, Phys. Rev. B 2009, 79, 041405.

[29] Z. Y. Li, N. P. Young, M. Di Vece, S. Palomba, R. E. Palmer, A. L. Bleloch, B. C. Curley, R. L. Johnston, J. Jiang, J. Yuan, Nature 2008, 451, 46.

[30] K. Tay, F. Bresme, Mol. Simulat. 2005, 31, 515.

[31] R. Singh, J. W. Lillard Jr., Exp. Mol. Pathol. 2009, 86, 215. 
[32] A. Boker, Y. Lin, K. Chiapperini, R. Horowitz, M. Thompson, V. Carreon, T. Xu, C. Abetz, H. Skaff, A. D. Dinsmore, T. Emrick, T. P. Russell, Nat. Mater. 2004, 3, 302.

[33] D. Moinard-Checot, Y. Chevalier, S. Briancon, L. Beney, H. Fessi, J. Colloid Interf. Sci. 2008, $317,458$. 

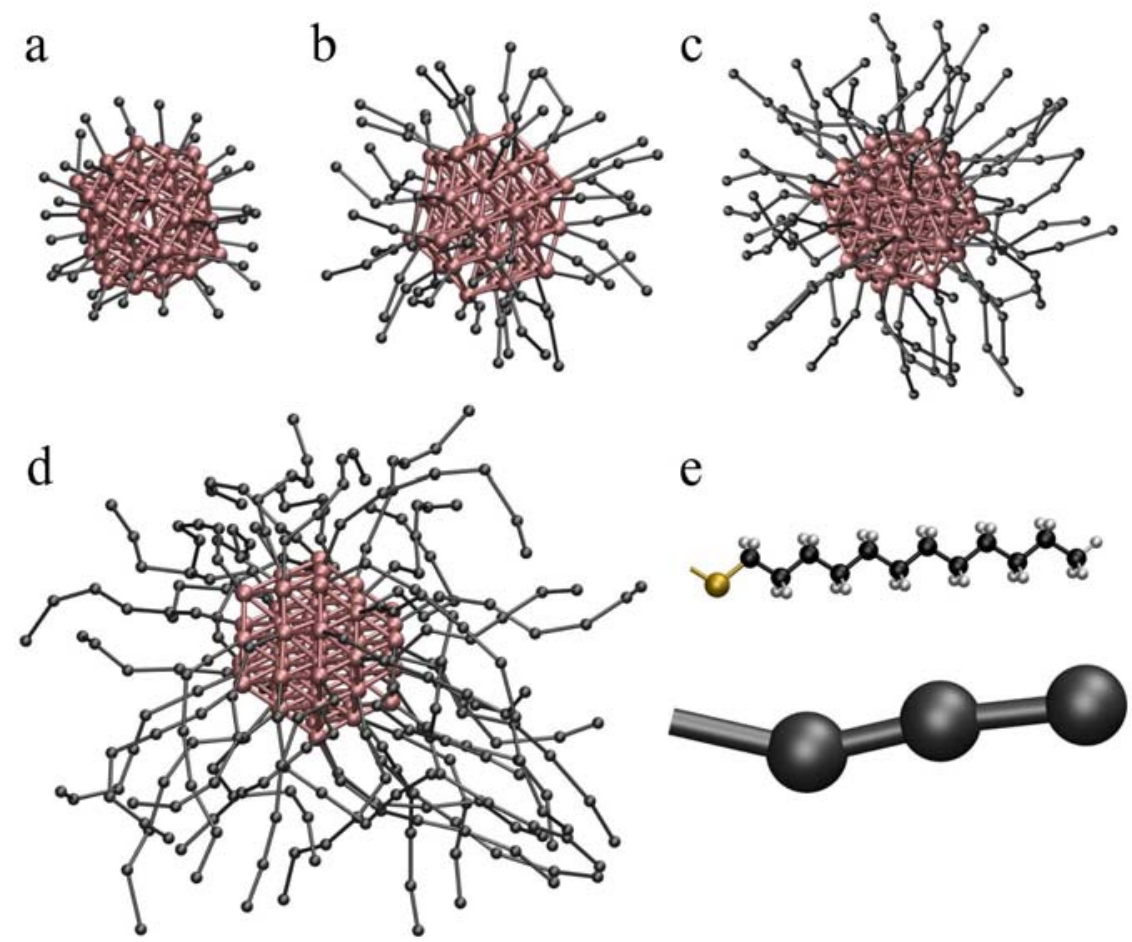

e
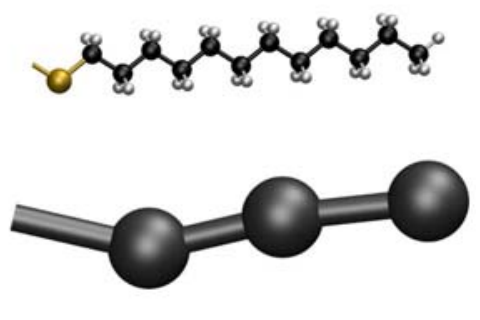

FIG. 1: Coarse-grained gold nanoparticles with core radius of $r_{\text {core }} \approx 0.8 \mathrm{~nm}$ capped with alkanethiol ligands of different lengths. (a) 1-bead ligand: $l_{\text {ligand }} \approx 0.5 \mathrm{~nm}$ and $R_{L C}=0.62$, (b) 2-bead ligand: $l_{\text {ligand }} \approx 0.9 \mathrm{~nm}$ and $R_{L C}=1.12$, (c) 3-bead ligand: $l_{\text {ligand }} \approx 1.3 \mathrm{~nm}$ and $R_{L C}=1.62$, (d) 6-bead ligand: $l_{\text {ligand }} \approx 2.6 \mathrm{~nm}$ and $R_{L C}=3.24$. (e) Dodecanethiol ligand is coarse-grained as three $\mathrm{C}_{1}$-type beads. 

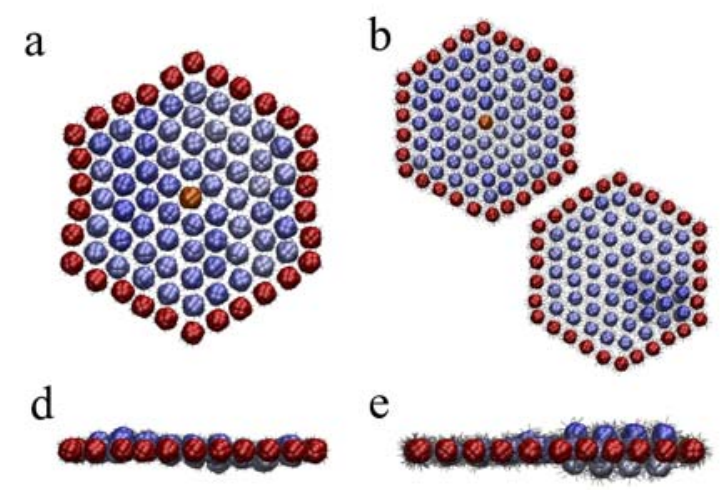

$\mathrm{e}$
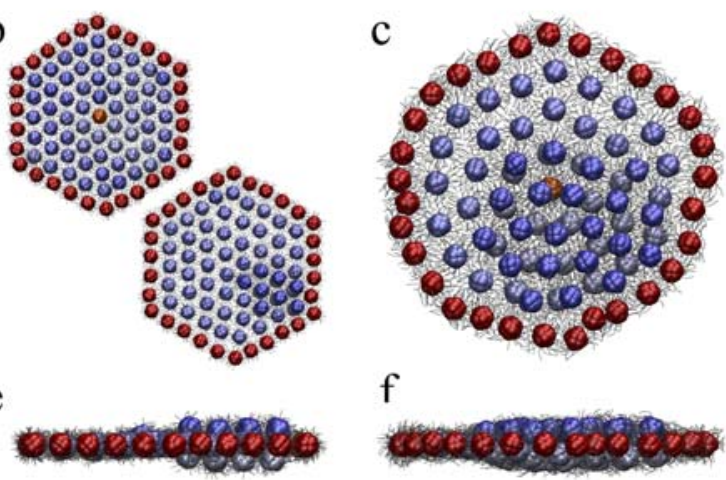

f
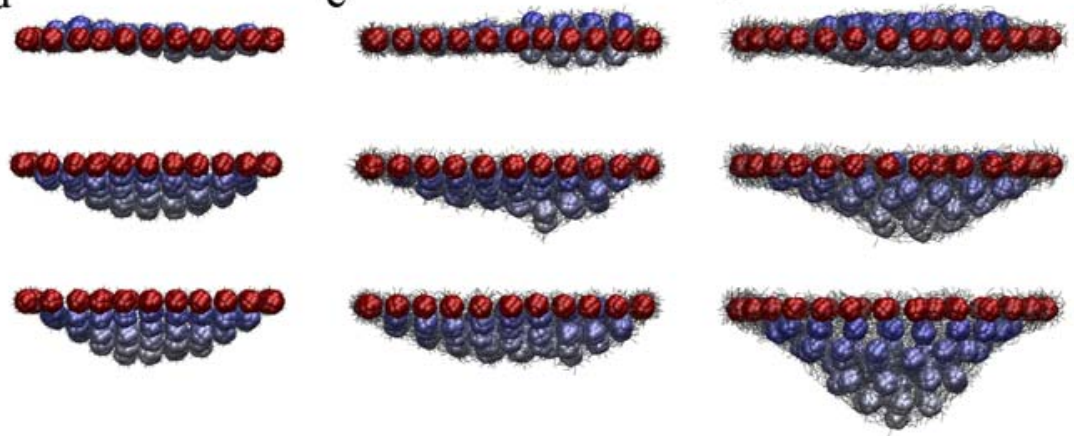

FIG. 2: (a-c) Relaxed membranes formed by the NPs with 1-3-bead ligands, respectively. Two membranes are shown for the system with 2-bead ligands, where one is a monolayer and the other is a partial double-layer (smaller scale). Central beads of NPs on the edge of the membrane (in red) are fixed while the rest of the NPs are free. (d) The 1-bead membrane under different level of stretching. When $\sigma=0.0,25.4$, and $44.6 \mathrm{pN} / \mathrm{nm}^{2}, D_{z}=0.0,-0.37$, and -0.45 respectively. (e) The 2-bead membrane monolayer under $\sigma=0.0,26.5$, and $50.0 \mathrm{pN} / \mathrm{nm}^{2}$ with $D_{z}=0.0,-0.26$, and -0.33 respectively. (f) The 3 -bead membrane when $\sigma=0.0,46.8$, and $105.5 \mathrm{pN} / \mathrm{nm}^{2}$ with $D_{z}=0.0,-0.34$, and -0.65 . 


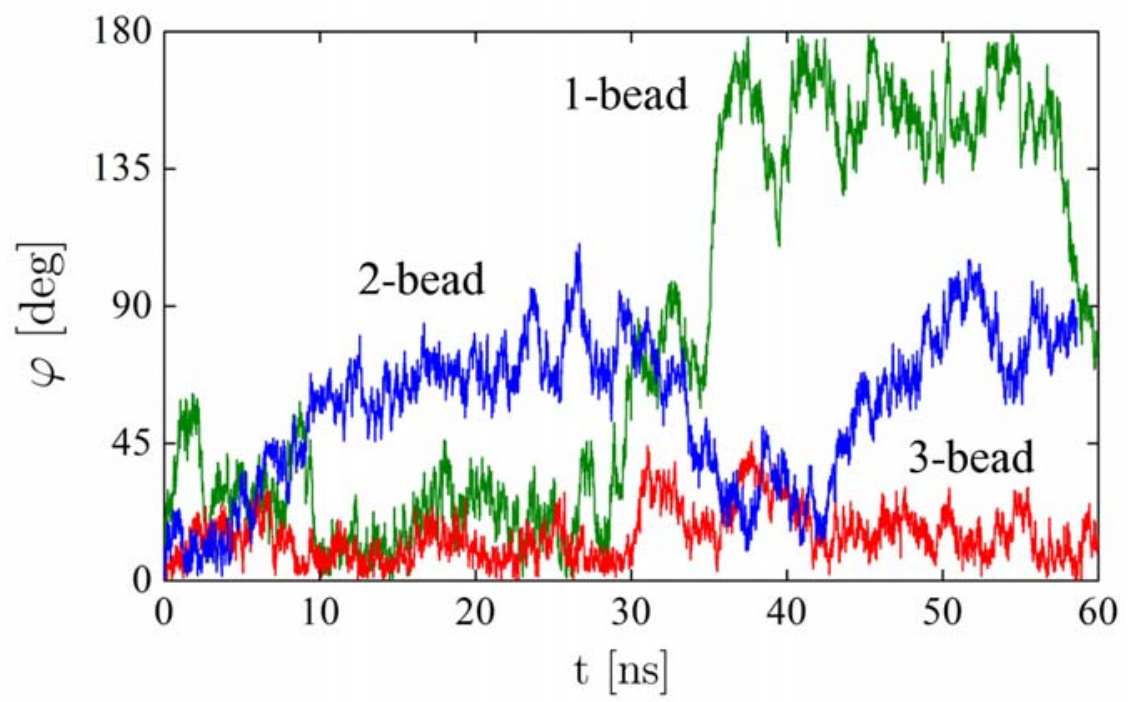

FIG. 3: Rotational motion of the NP (highlighted in Fig. 2) placed at the center of the 1-3-bead relaxed membranes. The random NP-rotation with the azimuthal angle $\varphi$ is very different in the three systems. 


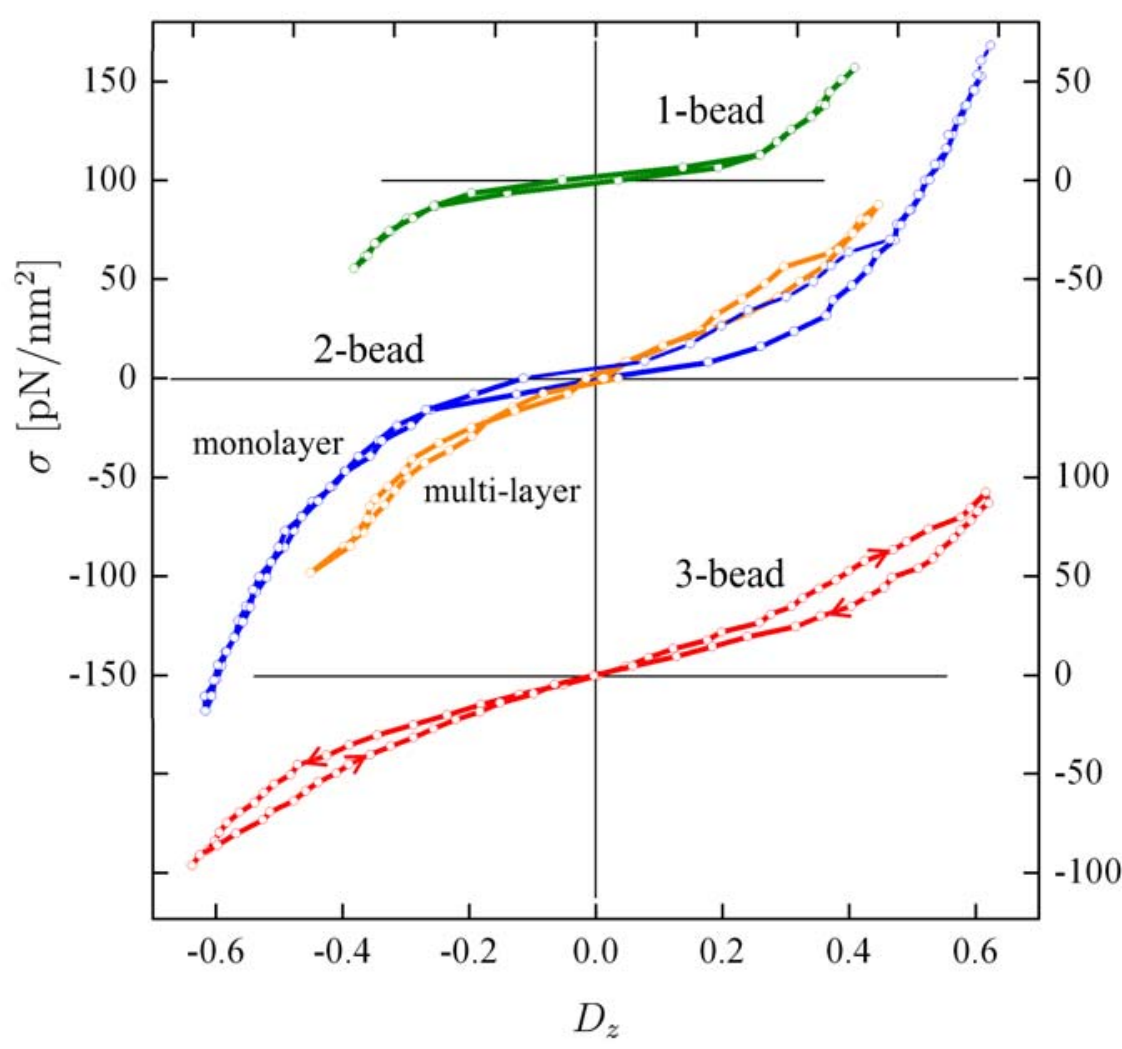

FIG. 4: Stress-strain curves for the stretching of NP-membranes with $R_{L C}=0.62$, (top), $R_{L C}=$ 1.12 (middle), and $R_{L C}=1.62$ (bottom). $\sigma$ is the applied stretching force per unit area of the membrane, $D_{z}$ is the average NP-displacement divided by the average radius $R_{m}$ of the membrane. Regions with loops reflect multilayer formation. The scales for 1-bead and 3-bead are placed on the right while the scale for 2-bead is on the left. 

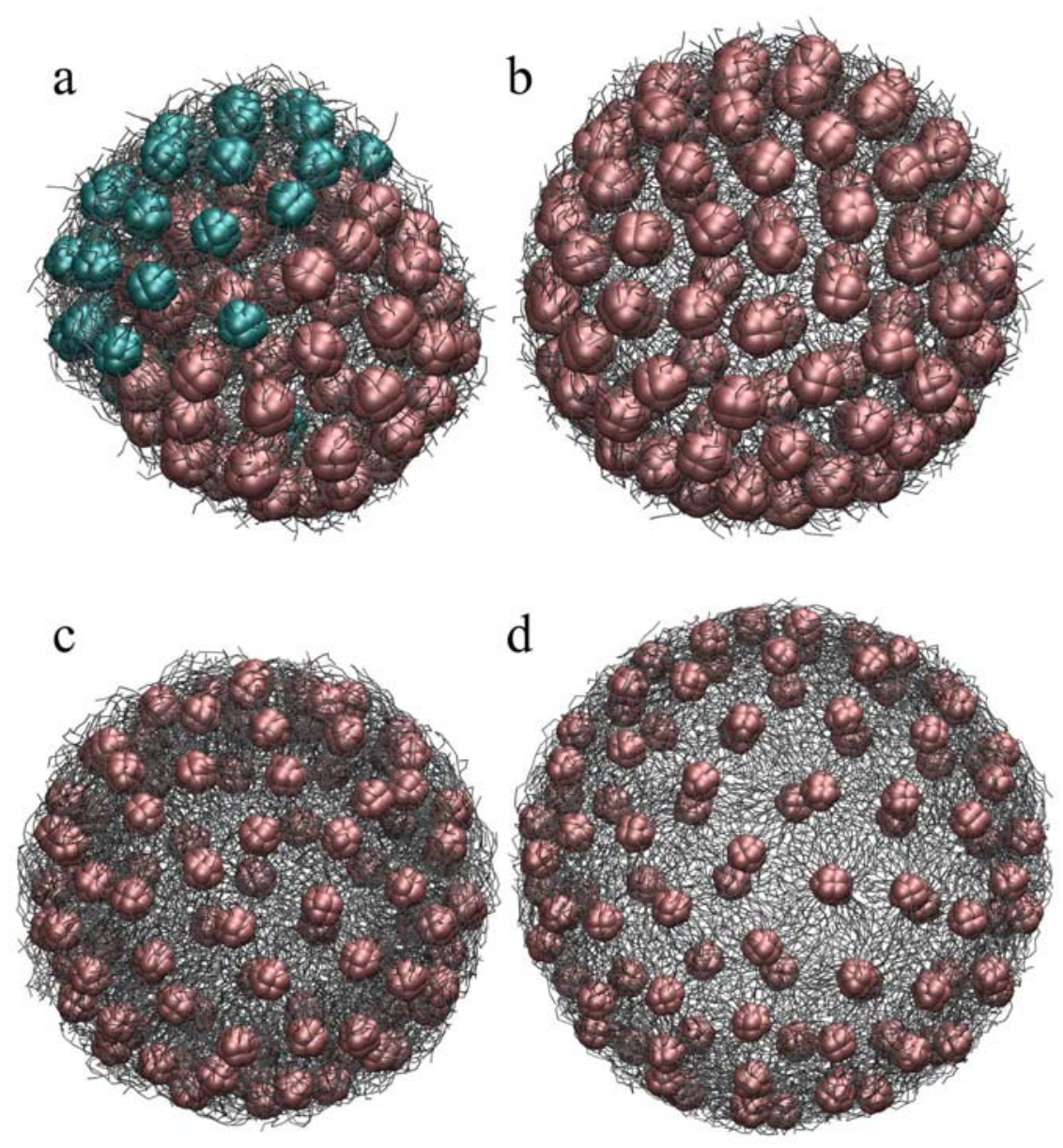

FIG. 5: Spherical capsules formed by 96 NPs with 3-bead and 6-bead ligands. Capsule with 3-bead ligands: (a) partial double-layer is formed for $R_{s}=5 \mathrm{~nm}$ and (b) it becomes a monolayer for $R_{s}=7$ nm. Capsule with 6-bead ligands: (c) monolayer for $R_{s}=8 \mathrm{~nm}$ becomes fully stretched (d) for $R_{s}=10 \mathrm{~nm}$. 


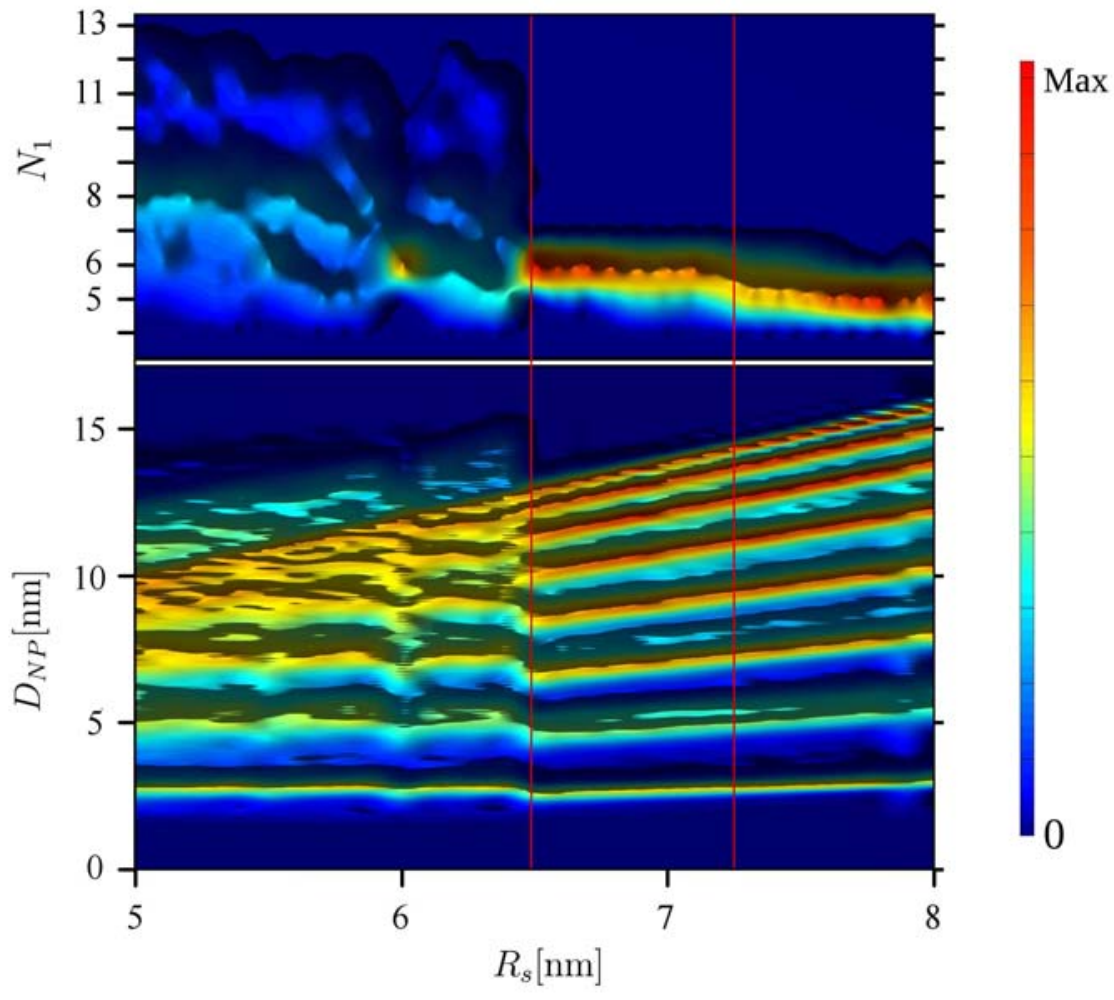

FIG. 6: Correlations of NP-distances in the capsule with 3-bead ligands as a function of the radius $R_{s}$ : (top) the average number of 1 st neighbors, $N_{1}$, and (bottom) the average distances to all the other NPs, $D_{N P}$. Lighter regions in the $2 \mathrm{D}$ maps signify higher probabilities. The red line on the right marks the transition from 5 1st neighbor to 6 1st neighbors. The red line on the left marks the point of double layer transition. The scale on the right show the relative heights of regions with different colors; all the distributions are normalized when integrated in the vertical direction. 


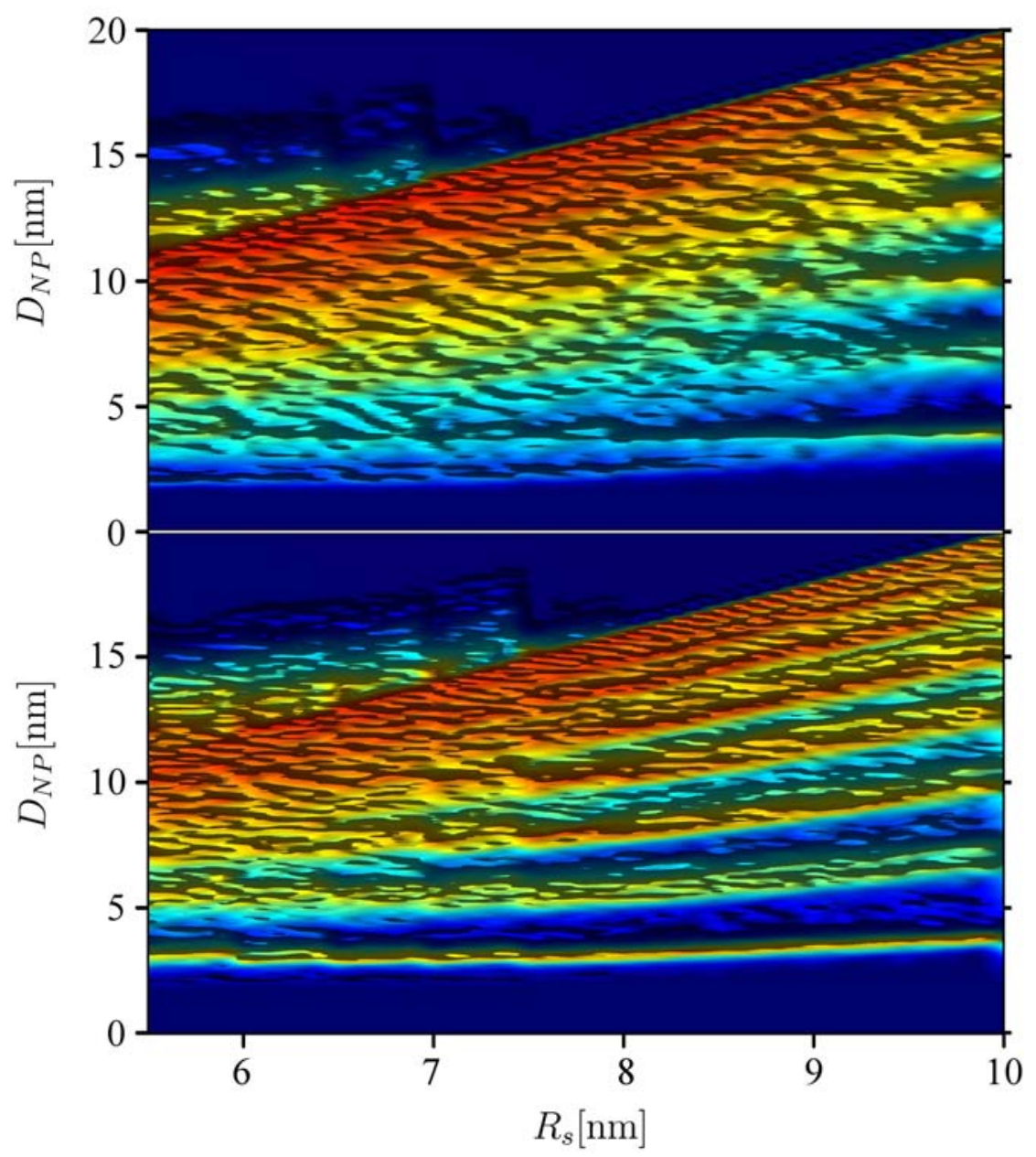

FIG. 7: The average distance between NPs, $D_{N P}$, for the NP-capsule with 6-bead ligands (tetracosanethiol): (top) non-modified ligands and (bottom) modified ligands. 


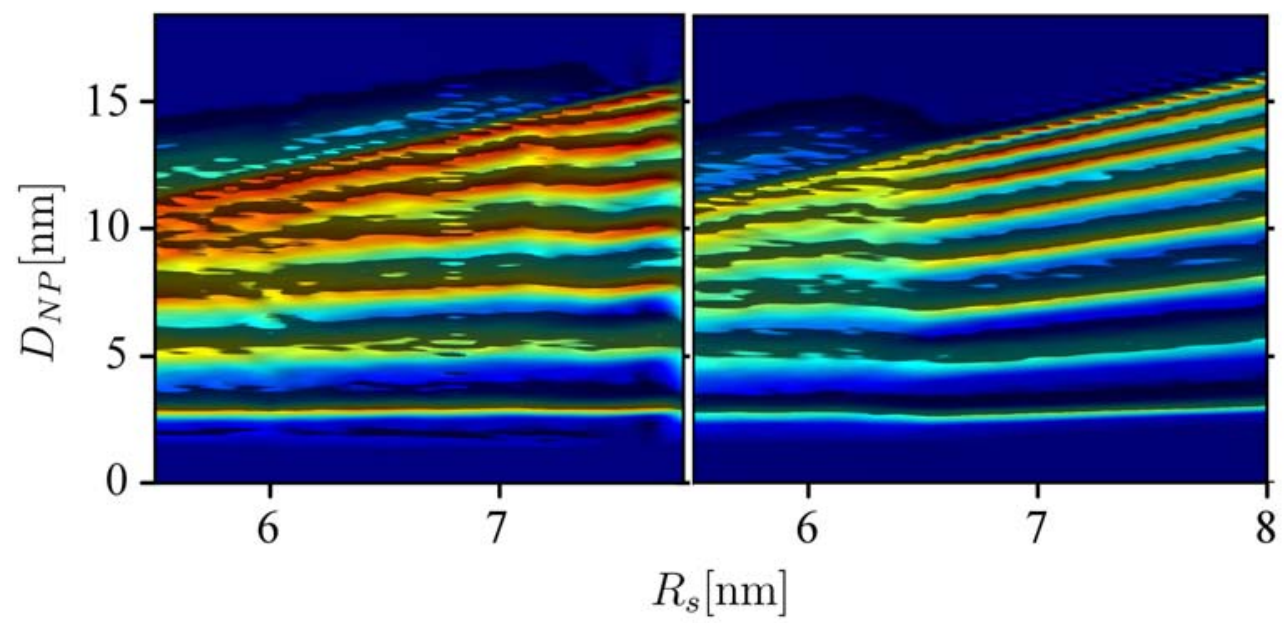

FIG. 8: The average distance between NPs, $D_{N P}$, for the NP-capsule with 3-bead ligands: (left) $10 \%$ defective NPs and (right) addition of 1,000 dodecane molecules. 

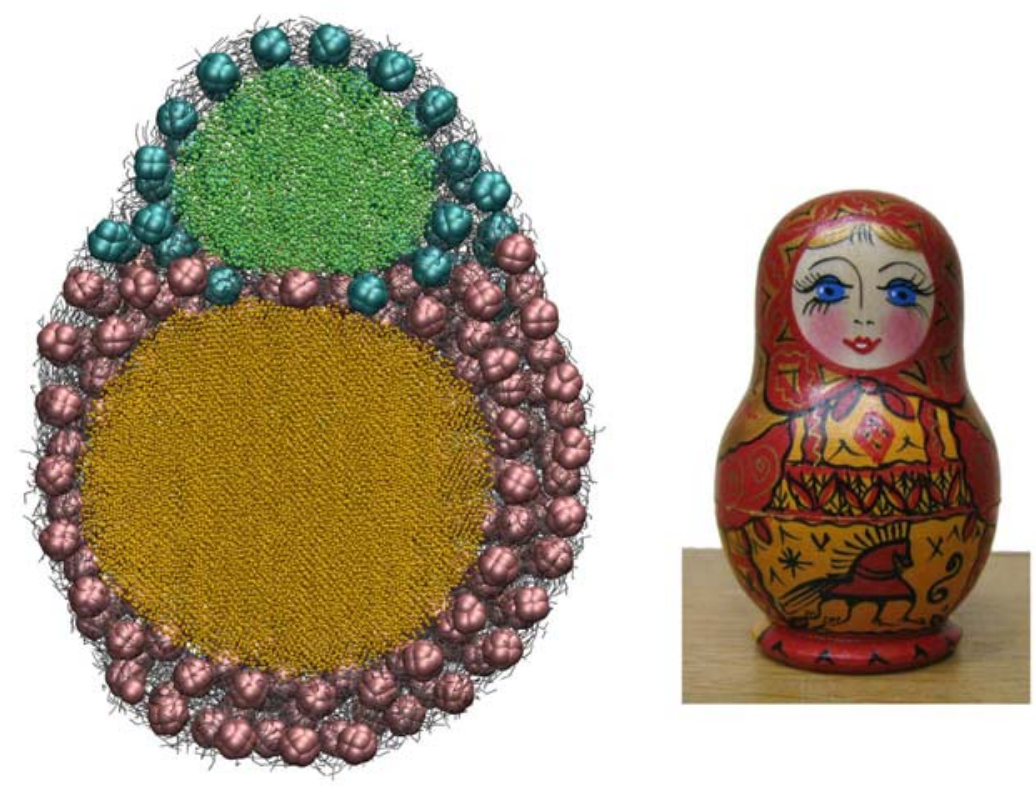

FIG. 9: (left) Sectional view of two water filled containers. (right) Shape comparable to a matryoska. 